\title{
Supplementary Orienting cellulose nanocrystal functionalities Information (SI) tunes the wettability of water-cast films
}

\author{
Charles Bruel, ${ }^{a}$ Salomé Queffeulou, Pierre J. Carreau, Jason R. Tavares, \\ and Marie-Claude Heuzey ${ }^{b}$
}

This supplementary information (SI) includes a nomenclature of the abbreviations, symbols, and variables used in the main text. It also gathers: extended protocols; CNC structural characterization (purity, Zeta-potential, dimensions, crystallinity, and cross-sectional shape); the methodology that was used to minimize kinetic and roughness-induced contact angle hysteresis; considerations over the surface tension parameters of the fluid and over the influence of surface charges (ionic dissociation) on the mechanisms proposed in the main text; and the description of a typical advancing/receding contact angle (ARCA) experiment. Finally, Table SI 5 summarizes ARCA results and their statistical analysis.

\section{Extended protocols}

Transmission electron microscopy: A Jeol JEM-2100F field emission electronic microscope performed imaging (acceleration voltage of $200 \mathrm{kV}$ ) of nanocrystals deposited on copper-based TEM grids (CF200-Cu, Electron Microscopy Sciences) coated by 5-6 $\mathrm{nm}$ of pure carbon. The grids were dipped in an aqueous CNC suspension diluted at $10 \mu \mathrm{g}_{\mathrm{CNC}} / \mathrm{mL}$ and dried for $24 \mathrm{~h}$ under the hood. TEM images were analyzed manually using the software ImageJ.11 Standard deviations are reported.

Elemental analysis: A Tabletop Hitachi TM3030+ scanning electron microscope performed quantitative elemental analysis on the oven-cast thin films through energy-dispersive X-ray spectroscopy (EDX). Acquisition was achieved for $60 \mathrm{~s}$ on three different locations with an electron beam generated from a $15 \mathrm{kV}$ source. Standard deviations were below $\sim 1 \%$ and are not reported.

Zeta-potential: A Malvern Zetasizer Nano-ZS, operated with a DTS1070 cell, performed Zeta-potential measurements on dilute CNC suspensions ( $3 \mathrm{mg}_{\mathrm{CNC}} / \mathrm{mL}$ ). Zeta-potential was determined based on 3 successive measurements (10 runs of $15 \mathrm{~s}$ each) performed on 3 different samples (total of 9 measurements). The standard deviation is reported.

X-ray diffraction: A X'pert instrument (Philips) performed Xray diffraction (XRD) on the CNC spray-dried powders and on the oven-cast thin films. It operated with $\mathrm{Cu} \mathrm{K} \alpha$ radiations (wave-

Research Center for High Performance Polymer and Composite Systems (CREPEC), Chemical Engineering Department, Polytechnique Montreal, P.O. Box 6079, Stn CentreVille, Montreal, QC H3C 3A7, Canada.

${ }^{a}$ E-mail: charles.bruel@polymtl.ca

${ }^{b}$ E-mail: marie-claude.heuzey@polymtl.ca
Table SI. 1 Nomenclature. As a general rule for variables, while superscripts point to the substrate characterized by the variable, subscripts refers to variable specifications. Elements that appear in SI but not in the main text (such as crystallographic variables) are not listed.

\begin{tabular}{|c|c|}
\hline \multicolumn{2}{|c|}{ List of abbreviations and acronyms } \\
\hline AFM & Atomic force microscopy \\
\hline ARCA & Advancing/receding contact angle \\
\hline CA & Contact angle \\
\hline $\mathrm{CNC}$ & Cellulose nanocrystal \\
\hline DIM & Diiodomethane \\
\hline EDX & energy-dispersive X-ray spectroscopy \\
\hline EG & Ethylene glycol \\
\hline OC & Oven-cast \\
\hline SC & Spin-coated \\
\hline TEM & Transmission electron microscopy \\
\hline $\mathrm{XRD}$ & X-ray diffraction \\
\hline \multicolumn{2}{|c|}{ List of variables } \\
\hline$\delta$ & Hansen solubility parameter $\left(\mathrm{MPa}^{1 / 2}\right)$ \\
\hline$\gamma$ & Surface tension $\left(\mathrm{mN} \mathrm{m}^{-1}\right)$ \\
\hline$\theta$ & Contact angle $\left({ }^{\circ}\right)$ \\
\hline$\rho$ & Density $\left(\mathrm{g} \mathrm{cm}^{-3}\right)$ \\
\hline$\eta$ & Viscosity (Pa s) \\
\hline$\Delta E$ & Surface energy variations $\left(\mathrm{mJ} \mathrm{m}^{-2}\right)$ \\
\hline$D$ & Diameter (m) \\
\hline$f$ & Surface fraction $(0 \leq f \leq 1$, dimensionless) \\
\hline$H$ & Contact angle hysteresis $\left(=\theta_{\mathrm{a}}-\theta_{\mathrm{r}},{ }^{\circ}\right)$ \\
\hline$v$ & Rate of progression over a surface $\left(\mathrm{m} \mathrm{s}^{-1}\right)$ \\
\hline \multicolumn{2}{|c|}{ List of variables' superscripts } \\
\hline drop & Drop, applies to $D^{\text {drop }}$ \\
\hline 1 & liquid, applies to $\gamma^{1}, \rho^{1}, \eta^{1}, v^{1}$ \\
\hline nps & non-polar surfaces, applies to $\delta^{\mathrm{nps}}, \gamma^{\mathrm{nps}}, \theta^{\text {nps }}, f^{\mathrm{nps}}$ \\
\hline ps & polar surfaces, applies to $\delta^{\mathrm{ps}}, \gamma^{\mathrm{ps}}, \theta^{\mathrm{ps}}, f^{\mathrm{ps}}$ \\
\hline $\mathrm{s}$ & solid, applies to $\gamma^{s}$ \\
\hline $\mathrm{W}$ & water, applies to $\gamma^{\mathrm{W}}$ \\
\hline \multicolumn{2}{|c|}{ List of variables' subscripts } \\
\hline $\mathrm{a}$ & Advancing, applies to $\theta_{\mathrm{a}}$ \\
\hline comp & Composite, applies to $\theta_{\text {comp }}$ \\
\hline $\mathrm{r}$ & Receding, applies to $\theta_{\mathrm{r}}$ \\
\hline $\mathrm{D}$ & Dispersive, applies to $\delta_{\mathrm{D}}, \gamma_{\mathrm{D}}$ \\
\hline $\mathrm{H}$ & Hydrogen-bonding, applies to $\delta_{\mathrm{H}}$ \\
\hline $\mathrm{P}$ & Polar, applies to $\delta_{\mathrm{P}}, \gamma_{\mathrm{P}}$ \\
\hline $\mathrm{T}$ & Total, applies to $\delta_{\mathrm{T}}$ \\
\hline W & Wenzel, applies to $\theta_{\mathrm{W}}$ \\
\hline Y & Young, applies to $\theta_{\mathrm{Y}}$ \\
\hline \multicolumn{2}{|c|}{ Other symbols } \\
\hline$g$ & Gravitational force constant $\left(\sim 9.81 \mathrm{~m} \mathrm{~s}^{-2}\right)$ \\
\hline$N_{\mathrm{CA}}$ & Capillary number (dimensionless) \\
\hline$N_{\text {Bo }}$ & Bond number (dimensionless) \\
\hline$r_{\text {surf }}$ & Surface roughness coefficient (dimensionless) \\
\hline$R_{\mathrm{q}}$ & Standard deviation in terms of surface's heigth $(\mathrm{m})$ \\
\hline
\end{tabular}


length of $0.1542 \mathrm{~nm}$ ) that were generated at $50 \mathrm{kV}$ by a current of $40 \mathrm{~mA}$. The $2 \theta$ angle was varied from $5.01^{\circ}$ to $49.99^{\circ}$ at a step of $0.02^{\circ}$ per scan (scan time of $1 \mathrm{~s} /$ step).

Atomic force microscopy: An ICON Microscope (BrukerSanta Barbara, CA) performed atomic force microscopy (AFM) on the spin-coated and oven-cast films. Analyses were carried at room temperature in air in Peak Force Tapping mode operated at a scan rate of $1 \mathrm{~Hz}$ with silicon nitride cantilevers (model: ScanAsyst-Air from Bruker). Acquisition was assisted by a scan Asyst parameter optimization routine. The cantilever resolves height resolution within $0.2 \mathrm{~nm}$. Planar resolution is, however, limited by the geometry of its tip and falls in the range of $\sim 10 \mathrm{~nm}$.

\section{CNC structural characterization}

\section{Purity and surface charge}

The purity of our CNC samples and their level of sulfatation was assessed by an elemental analysis performed on oven-cast films through energy-dispersive X-ray (EDX) analysis. CNCs have a $\mathrm{S} / \mathrm{C}$ ratio of 0.0061 , which correspond to 3.6 sulfate half-ester groups per 100 anhydroglucose units according to the equation by Hamad and $\mathrm{Hu}^{2}$. These sulfate half-ester groups introduce negative charges at the surface of the nanocrystals that result in a Zeta potential of $-45.5 \pm 3.8 \mathrm{mV}$. The $\mathrm{O} / \mathrm{C}$ ratio is of 0.85 , which, once corrected for the oxygen atoms that belong to the sulfate half-ester groups, yields a value of $\sim 0.83$ for the unsulfated CNC backbone. This is because each sulfur is bonded to 4 oxygen atoms, three of which do not belong to cellulose backbone. It coincides with the theoretical $\mathrm{O} / \mathrm{C}$ ratio of 0.83 for cellulose chains and is indicative of a high level of purity: the presence of impurities, especially lignin, is expected to decrease this ratio since cellulose is the oxygen-richest component in plant cell walls. 3

\section{Dimensions}

TEM images displayed an obvious mix of individual CNC particles and agglomerates so that the differentiation may be hard to tell. Counting 250 CNCs bundles (without making any size discrimination) leads to an average length, $L_{\mathrm{CNC}}$, of $150 \mathrm{~nm}$ and an average diameter, $D_{\mathrm{CNC}}$, of $10.0 \mathrm{~nm}$ with respective standard deviations of $70 \mathrm{~nm}$ and $4.5 \mathrm{~nm}$. In our former work, where TEM grids were prepared by depositing and drying a drop on the surface, a bundle's length of $\sim 165 \mathrm{~nm}$ and a width of $\sim 13 \mathrm{~nm}$ were reported for the same CNCs. 4 Here, the grids were immersed in the suspension and withdrawn, resulting in a smaller thickness of liquid being deposited on the surface. That the protocol influences the average size of CNC bundles has already been reported. 5 This influence arises from the reagglomeration that takes place upon preparation of the TEM grids and illustrates the need to perform a more in-depth analysis such as the one published by Elazzouzi-Hafraoui et al. 6 . Therefore, the distribution in terms of width $\times$ length was plotted to discriminate agglomerates from individual CNC particles (Fig. SI 1). A peak appears in the range of $L_{\mathrm{CNC}} \approx 105-120 \mathrm{~nm}$ and $D_{\mathrm{CNC}} \approx 7-8 \mathrm{~nm}$. The smallest particles are, however, about $4-5 \mathrm{~nm}$ in diameter, which is in the range of what has been reported for plant-based $\mathrm{I}_{\beta}$ elementary microfibrils.7. 9

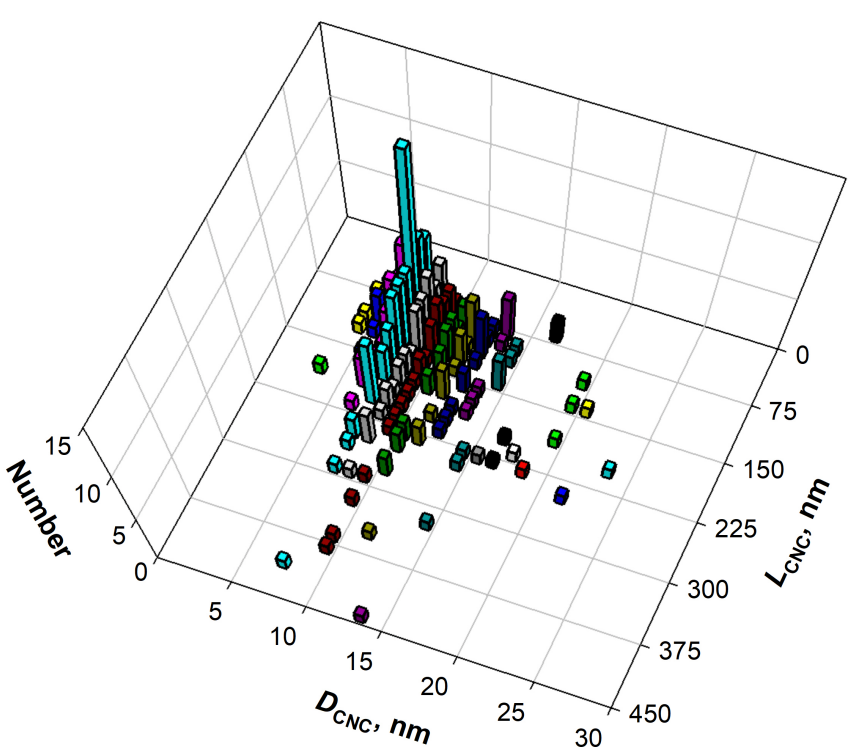

Fig. SI. 1 Length $\left(L_{\mathrm{CNC}}\right)$ times diameter $\left(D_{\mathrm{CNC}}\right)$ distribution of $\mathrm{CNC}$ bundles on TEM images based on an analysis with ImageJ.1 Each bar represents an interval of $15 \mathrm{~nm}$ for the length and $1 \mathrm{~nm}$ for the diameter. The height of a bar is proportional to the number of counts (out of 250) for the corresponding $L_{\mathrm{CNC}} \times D_{\mathrm{CNC}}$ interval. The disctribution is centered around a peak corresponding to $D_{\mathrm{CNC}} \approx 7-8 \mathrm{~nm}$ and $L_{\mathrm{CNC}} \approx 105-120 \mathrm{~nm}$, which are assumed to correspond to the dimensions of an individual nanocrystal.

\section{Cristallography}

CNC alignment in the films: Fig. 2a plots the X-ray diffraction spectra for both spray-dried CNC powders and oven-cast films. Spin-coated films were too thin to be analyzed properly. 5 main peaks, labeled accordingly to their Miller indices (hkl), 10 may be observed in cellulose $\mathrm{I}_{\beta}$ nanocrystal samples. They correspond to the lattice planes (110), (110), (012), (200), and (004); their respective peak maxima are expected at angles, $2 \theta$, of $15.1,16.8$, $21.0,22.8$, and $34.6^{\circ}$, respectively, $\underline{611}$ where $\theta$ is the Bragg angle. These 5 peaks appear in the spectrum of the powder. Note that the peak for the lattice plane (012) appears as a shoulder to the (200) peak. It is however interesting to note that peaks (012) and (004) disappear in the film spectrum. This is attributed to the alignment of the CNCs within the films: while CNCs are randomly oriented within spray-dried agglomerates, they align upon solvent casting in water. As a consequence, they lie in the film with their long axis parallel to the air/film interfaces. This is coherent with the AFM images presented in Fig. 1 of the main text. Elazzouzi-Hafraoui et al. 6 reported similar results by analyzing CNC films from their top side or from their cross-section: the peaks (012) and (004), visible from the cross sectional observation, disappeared in the analysis performed from the top side. Fig. 2 b plots the cross-section of cellulose $\mathrm{I}_{\beta}$ crystal cell and displays its lattice planes (schemes in background represent individual cellulose chains ${ }^{12}$ ).

Crystallinity index: Using Segal's equation (Eq. SI, 1, $\frac{14[15}{15}$ we estimated the cristallinity index, $C I$, of our sample to be of $\sim 81 \%$ 

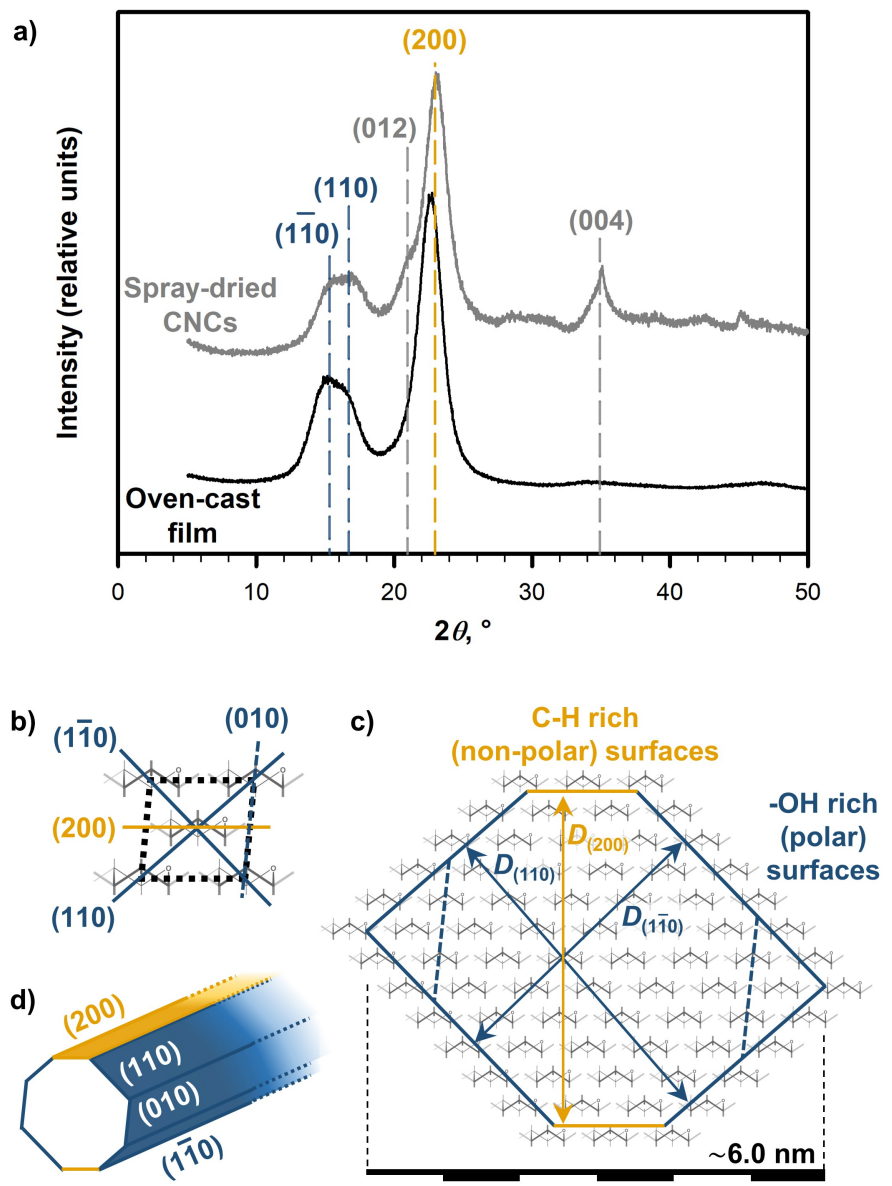

Fig. SI. 2 XRD spectra of spray-dried CNCs (top grey line) and ovencast films (bottom black line) (a). The spectrum is typical of cellulose $\mathrm{I}_{\beta}$ nanocrystals. 5 peaks corresponding to the lattice planes (110), (110), (012), (200), and (004) are visible on the spectrum of the spraydried sample which denotes a random orientation of the nanoparticles within the powder. The spectrum of the film, however, only shows the 3 peaks of the nanocrystal's cross-section: (110), (110) and (200), which points towards an alignment of the nanocrystals within the film. The corresponding cross-section of the crystalline cell unit is plotted in (b): hydroxyl groups rich lattice planes are plotted in blue, $\mathrm{C}-\mathrm{H}$ bond rich ones are in yellow. Molecule schemes, in background, each representing the cross-section of a cellulose chain viewed from its long-axis. In (c), the nanocrystal cross-sectional shape is triangulated from the XDRdetermined estimates of $D_{(110)}, D_{(1 \overline{1} 0)}$, and $D_{(200)}$. The presence of (010) surfaces (dashed blue lines), often hypothesized for CNCs, $\frac{13}{13}$ cannot be proven by XRD. However, this bears little consequences on the overall chemistry of the nanocrystal. The octagonal cross-sectional shape considered in this work is displayed in (d).

in the powder and of $\sim 87 \%$ in the films. Segal's formula is an empiric equation that relies on the determination of the maximum intensity of the peak (200), $I_{(200)}$, and of the maximum intensity of the amorphous peak, $I_{\mathrm{AM}}$. The latter is measured as the minimum observed between (110) and (200) peaks.

$$
C I=100 * \frac{I_{(200)}-I_{\mathrm{AM}}}{I_{(200)}}
$$

This slight increase in $C I$ between the powder and the film may be attributed to the disappearance of the peak (012), that creates a shoulder in the (200) peak of the powder. This probably helps in
Table SI. 2 Parameters of the crystallographic analysis. $\lambda_{\mathrm{XRD}}=0.1542 \mathrm{~nm}$ and $K=0.9$ were considered to calculate $D_{(\mathrm{hkl})}$ using Scherrer's equation (Eq. SI 2).

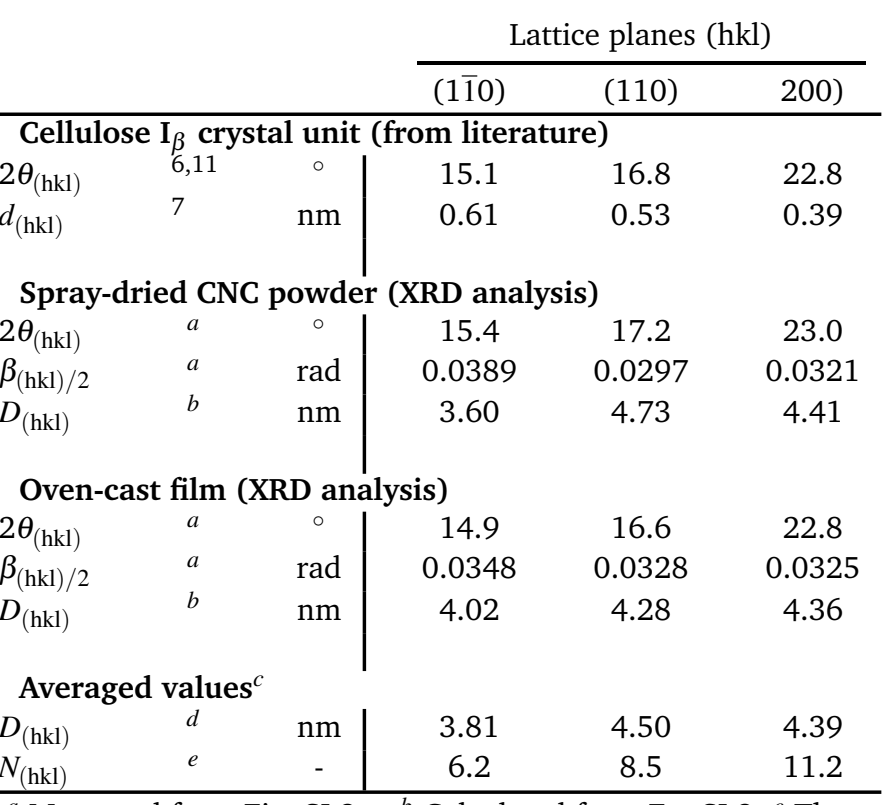

${ }^{a}$ Measured from Fig. SI 2 a. ${ }^{b}$ Calculated from Eq. SI $2{ }^{c}$ These average values were used to plot the cross-sectional shape displayed in Fig. SI 2 c. ${ }^{d}$ Average of $D_{(\mathrm{hkl})}$ measured from the spray-dried powder and the oven-cast thin films. ${ }^{e}$ Calculated from Eq. SI 3

determining $I_{\mathrm{AM}}$ by clearing the gap between the (110) and (200) peaks. A real increase in cristallinity cannot, however, be totally ruled out: in their model for the cross section of $\mathrm{I}_{\beta}$ nanocrystals, Ding and Himmel ${ }^{9}$ proposed that surface chains, being influenced by their environment, are only para-crystalline. It is thus possible that solvent casting favors the overall crystallinity of the sample by providing an optimized vis-à-vis to surface chains, thus limiting their motility. Indeed, solvent casting enables a strong ordering between CNC particles through an evaporation-induced self-assembly mechanism. 16

Cross-sectional shape Through a deconvolution of the peaks, XRD data also enables for an estimation of the apparent crystallite size, $D_{(\mathrm{hkl})}$, using Scherrer's equation (Eq. SI 2] 611$]$

$$
D_{(\mathrm{hkl})}=K * \lambda_{\mathrm{XRD}} /\left(\beta_{(\mathrm{hkl}) / 2} * \cos \left(\theta_{(\mathrm{hkl})}\right)\right)
$$

Its parameters are the angular width of the (hkl) peak midheight, $\beta_{(\mathrm{hkl}) / 2}$, the Bragg angle of its maximum, $\theta_{(\mathrm{hkl})}$, and the wavelength of the XRD beam, $\lambda_{\mathrm{XRD}}(=0.1542 \mathrm{~nm}$ in our case). Note that $\beta_{(\mathrm{hkl}) / 2}$ needs to be expressed in radiant. $K$ is a dimensionless shape factor, whose typical value is between 0.9 and 1 . $K=0.9$ was considered as a measurement of $D_{(\mathrm{hkl})}$ lower bound. Three values, each corresponding to a lattice plane, may hence be calculated: $D_{(1 \overline{1} 0)}, D_{(110)}$, and $D_{(200)}$. No large deviations could be observed between the values derived from the powder spectrum and those derived from the film: their average was thus considered. $D_{(110)}, D_{(1 \overline{1} 0)}$, and $D_{(200)}$ were hence estimated at 4.5, 3.8, and $4.4 \mathrm{~nm}$, respectively. Table SI 2 summarizes all the param- 
eters relevant to the peak deconvolution and Fig. 2. c shows the triangulation of a nanocrystal cross section from $D_{(\mathrm{hkl})}$ values.

Knowing the parameters of a cellulose $\mathrm{I}_{\beta}$ cell unit $\left(a=0.778 \mathrm{~nm}, b=0.820 \mathrm{~nm}, c=1.038 \mathrm{~nm}, \gamma=96.5^{\circ}\right)^{10}$, it is possible to calculate the $d$-spacings, $d_{(\mathrm{hkl})}$, between lattice planes: $d_{(1 \overline{1} 0)}(0.61 \mathrm{~nm}), d_{(110)}(0.53 \mathrm{~nm})$, and $d_{(200)}(0.39 \mathrm{~nm}) .7$ From there, the number of cellulose chains, $N_{(\mathrm{hkl})}$ along each crystallographic plane may be calculated from the ratio between $D_{(\mathrm{hkl})}$ and $d_{(\mathrm{hkl})}\left(\right.$ Eq. SI 3). $N_{(\mathrm{hkl})}$ values are not integers, probably due to a combination of sample polydispersity and method uncertainty. Indeed, cross-sectional shape determination is a multi-step process whose uncertainty increases at each stage: XRD data acquisition, peak deconvolution, shape factor $K$. Cellulose chains are drawn in background of Fig. SI 2 c.

$$
N_{(\mathrm{hkl})}=\frac{D_{(\mathrm{hkl})}}{d_{(\mathrm{hkl})}}
$$

Hence, the cross-sectional shape that can be derived from XRD is hexagonal and predict the existence of (200) surface for CNCs (Fig. SI 2 c). In terms of surface area, and despite the uncer-

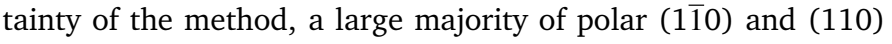
surfaces is expected. This is coherent with CNCs being reported as displaying a primarily hydrophilic behavior. 12 The presence of small (200) surfaces however comfort expectations that CNCs, as amphiphilic particles, should be able to display some preferential orientations at the air/water interface. It is also worth noting that the projected diameter of the nanocrystal found by XRD is, indepdantly of the shape factor ( $K=0.9$ or 1$)$ smaller than that determined through TEM for individual nanocrystals $(\sim 7-8 \mathrm{~nm})$. Previous works also reported such a difference.6]18

Literature often considers the presence of (010) surfaces (dashed blue lines on Fig. SI 2 b\&c), which provide an octagonal shape to the nanocrystals. 13 Their presence cannot be proven through XRD due to the lack of a (010) peak that would allow an estimation of $D_{(010)}$. However, this bears little consequences on the expected behavior of CNC particles: (010) lattice planes, when exposed, come to the detriment of (110)\&(110) surfaces with which they share the same hydroxyl rich chemistry. The crucial part is that, whatever the shape hypothesis (hexagonal or octagonal), CNCs keep displaying a majority of hydroxyl groups rich surfaces that are combined with a minority of $\mathrm{C}-\mathrm{H}$ bonds rich ones. This work considered octagonally-shaped nanocrystals (plotted in Fig. SI 2d) in the main text to abide by the convention found in most references on the interfacial behavior of cellulose nanocrystals in Pickering emulsions. 13

\section{Model applicability}

The following sections summarize the precautions that were taken in order to make non-surface tension related phenomena negligible and to minimize kinetic and surface roughness-induced hysteresis. This ensures that experimental data can be fitted with the model presented in Eq. 7 of the main text. The influence of liquid surface tension parameters on model predictions and of ionic dissociation on the energies calculated in the main text (Fig. 4) are also discussed.

\section{Capillary and Bond numbers}

The ratio between the hydrodynamic forces (viscosity of the liquid, $\eta^{1}$, times its rate of progression over the surface, $v^{1}$ ) and the surface tension forces of the liquid, $\gamma^{1}$, is defined as the capillary number, $N_{\mathrm{Ca}}$ (Eq. 4). In our case, the upper bound of $v^{1}$, which is controlled by the rate at which the liquid is injected/withdrawn, was measured at $\sim 0.05 \mathrm{mms}^{-1}$. It corresponds to a $N_{\mathrm{Ca}}$ of $\sim 2 \times 10^{-5}$ for EG and $\sim 3 \times 10^{-6}$ for DIM, a range at which hydrodynamic forces can be neglected. According to Strobel and Lyons 19 , $N_{\mathrm{Ca}}<10^{-5}$ generally ensures that the CA measured at the moving three-phase boundary is equal to that measured just after it stops, i.e. that it corresponds to a thermodynamic state. Overall, this condition was verified during our experiments.

$$
N_{\mathrm{Ca}}=\frac{\eta^{1} v^{1}}{\gamma^{1}}
$$

The ratio between the gravitational forces (liquid density, $\rho^{1}$, times gravitational acceleration, $g$, times the square of a characteristic length, $D^{\text {drop }}$ ) and the surface tension forces, $\gamma^{l}$, is defined as the Bond number, $N_{\text {Bo }}$ (Eq. 5). The upper bound of $D^{\text {drop }}$ was measured below $5 \mathrm{~mm}$ in all circumstances. It means that $N_{\mathrm{Bo}}$ remained below $\sim 6 \times 10^{-3}$ for EG and $\sim 2 \times 10^{-2}$ for DIM.

$$
N_{\text {Bo }}=\frac{\rho^{1} g\left(D^{\text {drop }}\right)^{2}}{\gamma^{\mathrm{l}}}
$$

$N_{\mathrm{Ca}}$ and $N_{\mathrm{Bo}}$ values prove that surface tension largely dominates (by 2 to 3 orders of magnitude) the force balance in our systems.

\section{Minimization of kinetic hysteresis}

Selecting an appropriate probe liquid is the main step that may be taken in order to minimize kinetic hysteresis.20121 Considering our aim, at least two solvents must be selected: one predominantly polar and the other non-polar. Their surface tension, $\gamma^{l}$, must be in the same range or greater than that of the solid in order to obtain non-zero contact angles. Van Oss et al .22 proposed that targeting a liquid surface tension above $\sim 44 \mathrm{mN} \mathrm{m}^{-1}$ was a safe threshold to carry the analysis of polar surfaces. Water $\left(\gamma^{1}=72.8 \mathrm{mN} \mathrm{m}^{-1}\right)$, glycerol $\left(64 \mathrm{mN} \mathrm{m}^{-1}\right)$, formamide ( $58 \mathrm{mN} \mathrm{m}^{-1}$ ), ethylene glycol (EG, $48 \mathrm{mN} \mathrm{m}^{-1}$ ), and dimethylsulfoxide (DMSO, $43.5 \mathrm{mN} \mathrm{m}^{-1}$ ), are the most common polar liquids. The threshold is hard to clear for non-polar liquids and in practice diiodomethane (DIM, $50.8 \mathrm{mN} \mathrm{m}^{-1}$ ) or $\alpha$ bromonaphtalene $\left(44.4 \mathrm{mN} \mathrm{m}^{-1}\right)$ are selected. $\frac{22}{2}$ They are two of the few non-polar liquids suitable for the analysis of polar surfaces.

In terms of kinetic hysteresis, the liquid must have a high boiling point (a criteria met by all the aforementioned solvents) to minimize evaporation and a low chemical affinity for the surface. Based on our previous manuscript on the chemical affinity of CNCs for solvents, $\frac{12}{12}$ water, formamide, and DMSO are unsuitable: their chemical affinity for CNCs is too strong. This causes a near instantaneous swelling of the film that was confirmed by performing dewetting tests in which these liquids failed to recede properly. The swelling was significant enough to be detected by 
the camera of the optical goniometer. It only left glycerol and EG as potential candidates. However, the high viscosity of glycerol (nearly two decades larger than that of EG) makes it hard to use for a dynamic CA experiment: it significantly increases the duration of the test, thus increasing the likelihood of kinetic phenomena (such as swelling) taking place. Previous investigations indeed suggested that any polar solvent may, up to a certain point, swell CNC thin films. ${ }^{23}$ Keeping the overall duration of each test within a few minutes is thus a top-priority and EG was selected as our polar probe liquid. Despite these precautions to select the polar liquid that causes the fewer swelling to the film, dewetting tests carried with EG remained difficult to analyze (see section "Wettability results and uncertainties" in SI). Regarding the non-polar probe solvent, DIM was selected for its higher surface tension and its low chemical affinity for CNCs.

\section{Thermodynamic hysteresis}

Thermodynamic hysteresis arises from a combination of chemical and topographical heterogeneities at the surface of the sample. 2425 Since measuring thermodynamic hysteresis caused by chemical heterogeneities is precisely the focus of this work, surface roughness needs to be controlled. Roughness influences contact angle measurements in at least 3 ways in drop-based methods. 24 Macroscale roughness (defined as being visible with the instrument's camera) favors intrumental error by creating an uncertainty on the sample baseline. At smaller scale, it turns the theoretical Young contact angle into an apparent ("Wenzel") contact angle. Finally, it favors the transition from an homogeneous (liquid in contact with the surface) to an heterogenous wetting regime, in which the liquid is only partially in contact with the sample due to some air bubbles remaining trapped in surface cavities. ${ }^{24}$ Estimating, and eventually minimizing, surface roughness is thus critical in making sure that what is measured is the real (by opposition to "apparent") contact angle of the drop in direct contact with the surface (homogeneous regime). Only in these circumstances may the results be interpreted in terms of surface tension of the substrate.

Macroscale roughness is expected to be nearly null for CNC films, which can be confirmed from their cross-sectional image observed with the optical goniometer: it eliminates any instrumental uncertainty regarding the detection of the baseline. Mesoscale roughness was measured according to two metrics: $r_{\text {surf }}$, which is the ratio between the surface area of the film and its projected surface area (see Eq. 5 of the main text); and $R_{\mathrm{q}}$, which is defined as the standard deviation in term of sample height. Both were calculated based on AFM measurements operating in tapping mode over a $5 \times 5 \mu \mathrm{m}^{2}$ area: $r_{\text {surf }}$ and $R_{\mathrm{q}}$ were respectively smaller than 1.02 and $15 \mathrm{~nm}$ in all our samples. Microscale roughness was assessed by measuring the standard deviation in term of sample's height, $R_{\mathrm{q}}$, over flattened micrographs: the surface is corrected by a baseline (second order polynomial) that accounts for the mesoscale roughness ( $r_{\text {surf }}$ is not reported for these flattened surfaces). $R_{\mathrm{q}}$ ranged from 2.0 to $8.5 \mathrm{~nm}$ for all our sample, independently of the surface area analyzed, which is in the range of a single nanocrystal diameter, $D_{\mathrm{CNC}}(\sim 7-8 \mathrm{~nm})$.
This level of roughness remains large with regards to the size of a liquid molecule. However, being in the range of $D_{\mathrm{CNC}}$, it is as flat as may be hoped for a surface made of self-assembled nanoparticles and we did not try to reduce it further. Indeed, while there is no consensus on the level of flatness that a sample must meet for its thermodynamic hysteresis to become neglectable, 1926 it is commonly accepted that a roughness below $\sim 0.05-0.1 \mu \mathrm{m}$ is unlikely to yield a significant hysteresis. ${ }^{20}$ These results also highlight the lack of any cavities susceptible to trap air bubbles on the films. it is worth noting that the tip of the cantilever as a geometry roughly $10 \mathrm{~nm}$ wide. It means that any feature whose width would be smaller than that, such as potential interstices between the self-assembled nanocrystals, cannot be detected by AFM.

\section{Surface tension coefficients}

\section{Probe liquids coefficients}

One of the tedious aspect of the Owens-Wendt-Rabel-Kaelble (OWRK) model is the accurate partition of the surface tension between a dispersive and a polar component (Eq. 2 of the main text). Indeed, while the total surface tension is easy to determine and consistent across the literature, it splits differently as a function of the source. For instance, diiodomethane $\left(\gamma^{1}=50.8 \mathrm{mN} \mathrm{m}^{-1}\right)$ is often considered as a purely dispersive sol-

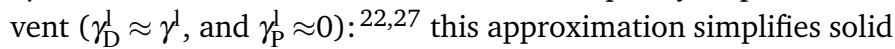
surface tension calculations from CA measurements. However, and like other liquid dihalogenomethanes $\left(\mathrm{CH}_{2} \mathrm{Cl}_{2}, \mathrm{CH}_{2} \mathrm{Br}_{2}\right)$, DIM has a small permanent dipole moment that results in a polar component for the surface tension. Hence this work considers the coefficients $\gamma_{\mathrm{D}}^{\mathrm{l}}=48.5 \mathrm{mN} \mathrm{m}^{-1}$, and $\gamma_{\mathrm{P}}^{\mathrm{l}}=2.3 \mathrm{mN} \mathrm{m}^{-1}$ for DIM as per Kaelble 28

Variations observed in the literature for ethylene glycol $\left(\gamma^{l}=48.0 \mathrm{mN} \mathrm{m}^{-1}\right)$ are more significant. $\gamma_{\mathrm{P}}^{1}$ vary from $19 \mathrm{mN} \mathrm{m}^{-129}$ to $29.3 \mathrm{mN} \mathrm{m}^{-130}$. This work considers an inbetween value of $\gamma_{\mathrm{P}}^{1}=25.4 \mathrm{mN} \mathrm{m}^{-1}$ (and $\gamma_{\mathrm{D}}^{1}=22.6 \mathrm{mN} \mathrm{m}^{-1}$ ). 31 This hypothesis bears no real influence on the $f^{\text {nps }}$ values determined for the spin-coated films through Eq. 7 of the main text. However, they shift the predictions by $\sim 0.05-0.07$ for the oven-cast films. Considering EG as a lowly polar liquid $\gamma_{\mathrm{P}}^{1}=19 \mathrm{mN} \mathrm{m}^{-129}$ slightly increases $f^{\mathrm{nps}}$ (0.49 instead of 0.42 ), while considering EG as a more polar liquid $\gamma_{\mathrm{P}}^{1}=29.3 \mathrm{mN} \mathrm{m}^{-1}$ slightly lowers it (0.37). Table SI 3 summarizes the influence of EG surface tension coefficients on model predictions for the ovencast films. Our conclusions are unaffected.

It is worth mentioning that using Eqs. 11\&12 of the main text, it is possible to estimate the surface tension coefficients of the probe liquids based on their HSP: $\underline{31}$

- DIM has HSP of $\{22.0 ; 3.9 ; 5.5\} \mathrm{MPa}^{1 / 2}$, which yields $\gamma_{\mathrm{D}}^{\mathrm{l}}=48.5 \mathrm{mN} \mathrm{m}^{-1}$ and $\gamma_{\mathrm{P}}^{\mathrm{l}}=2.2 \mathrm{mN} \mathrm{m}^{-1}$

- For EG $\left(\{17.0 ; 11.0 ; 26.0\} \mathrm{MPa}^{1 / 2}\right)$, it is $\gamma_{\mathrm{D}}^{\mathrm{l}}=26.5 \mathrm{mN} \mathrm{m}^{-1}$ and $\gamma_{\mathrm{P}}^{1}=23.9 \mathrm{mN} \mathrm{m}^{-1}$.

These predictions are close to the surface tension coefficients we considered (Table 3). 
Table SI. 3 Influence of the surface tension coefficients of EG on $f^{\text {nps }}$ predictions (Eq. 7 of the main text). Predictions are issued for advancing contact angle values of $\theta_{\mathrm{a}}=41.0 \pm 4.3^{\circ}$ for $\mathrm{EG}$ and $\theta_{\mathrm{a}}=46.1 \pm 1.2^{\circ}$ for DIM. Values in bold are those that are reported in the main text. "Ref." points to the references for the surface tension coefficients. Surface tensions are expressed in $\mathrm{mN} \mathrm{m}^{-1}$.

\begin{tabular}{cc|ccc|c} 
Liquid & Ref. & $\gamma$ & $\gamma_{\mathrm{D}}$ & $\gamma_{\mathrm{P}}$ & $f^{n p s}$ \\
\hline Diiodomethane & 28 & $\mathbf{5 0 . 8}$ & $\mathbf{4 8 . 5}$ & $\mathbf{2 . 3}$ & $\mathbf{0 . 4 4} \pm \mathbf{0 . 0 6}$ \\
Ethylene glycol & 29 & 48.0 & 29.0 & 19.0 & $0.49 \pm 0.09$ \\
- & 31 & $\mathbf{4 8 . 0}$ & $\mathbf{2 2 . 6}$ & $\mathbf{2 5 . 4}$ & $\mathbf{0 . 4 2} \pm \mathbf{0 . 0 8}$ \\
- & 30 & 48.2 & 18.9 & 29.3 & $0.37 \pm 0.07$ \\
\hline
\end{tabular}

Table SI. 4 Surface tension parameters for various cellulose $I_{\beta}$ nanocrystals' samples and surfaces as a function of the method of determination. Computer simulation enables for a discrimination between the various lattice planes (see Fig. SI 2 b). Our approach distinguishes polar surfaces (attributed to hydroxyl rich (110), (110), and (010) lattice planes) from non-polar ones (C-H bonds rich (200) lattice planes). 12 Other methodologies amalgamate CNC surfaces and report a single apparent surface tension whose value varies with the substrate morphology.

\begin{tabular}{|c|c|c|c|c|}
\hline & $\begin{array}{c}\gamma \\
\mathrm{mN} \mathrm{m}^{-1}\end{array}$ & $\begin{array}{c}\gamma_{\mathrm{D}} \\
\mathrm{mN} \mathrm{m}^{-1}\end{array}$ & $\begin{array}{c}\gamma_{\mathrm{P}} \\
\mathrm{mN} \mathrm{m}^{-1}\end{array}$ & Ref. \\
\hline \multicolumn{4}{|c|}{ Computer simulations } & \multirow{5}{*}{\begin{tabular}{|l|}
32 \\
32 \\
32 \\
\end{tabular}} \\
\hline (110) surfaces & 155 & - & - & \\
\hline (110) surfaces & 155 & - & - & \\
\hline (200) surfaces & 92 & - & - & \\
\hline \multicolumn{4}{|c|}{ Inverse gas chromatography } & \\
\hline Powder $^{a}$ & - & $45.6-68.5$ & - & \multirow[t]{2}{*}{33} \\
\hline \multicolumn{4}{|c|}{ Contact angle measurements } & \\
\hline \multirow[t]{2}{*}{ Oven-cast film ${ }^{b}$} & 68.9 & 40.9 & 28.0 & 34 \\
\hline & 69.5 & 42.0 & 27.5 & 35 \\
\hline Spin-coated film ${ }^{b}$ & 78.9 & 45.8 & 33.1 & 35 \\
\hline CNC pellets $^{b}$ & 62.1 & 40.9 & 21.2 & 34 \\
\hline \multicolumn{5}{|c|}{ This work: HSP modeling + CA measurements } \\
\hline Polar surfaces & $53.1^{c}$ & $30.7^{c}$ & $22.4^{c}$ & \multirow{4}{*}{ - } \\
\hline \multirow{3}{*}{ Non-polar surfaces } & $47.8-55.2^{d}$ & - & - & \\
\hline & $31.0^{c}$ & $28.0^{c}$ & $3.0^{c}$ & \\
\hline & $30.3-32.2^{d}$ & - & - & \\
\hline
\end{tabular}

${ }^{a}$ Measurements performed at $30^{\circ} \mathrm{C}$, the range corresponds to the difference between spray-dried and air-dried samples.

${ }^{b}$ Estimated from CA measurements through the Owens-Wendt-Rabel-Kaelble (OWRK) model. ${ }^{c} \gamma=\gamma_{D}+\gamma_{P}$, with $\gamma_{D}$ and $\gamma_{P}$ calculated from Eqs. $11 \& 12$ of the article, respectively.

${ }^{d}$ Range calculated from Eqs. $9 \& 10$ of the article

\section{CNC coefficients}

Table SI 4 provides a comparison between the surface tension coefficients determined in this work for CNCs and those reported in the literature. Previous characterization either failed to report the split of $\gamma$ in terms of dispersive and polar components, or to differentiate between CNC polar and non-polar surfaces. This is discussed in the main text (section "CNC's surface tension parameters").

\section{Influence of ionic dissociation}

Energy calculations presented in Fig. 4 of the article do not consider the enthalpy of dissociation between the sulfate half-ester groups of CNCs and their $\mathrm{Na}^{+}$counter ions (Eq. SI 6). However, upon CNC adsorbtion at the air/interface or upon CNC agglomeration, some stabilization brought by this counter-ion dissociation may be lost.

Based on elemental analysis, CNCs have a sulfur content of $\sim 0.75 \mathrm{wt} \%$ (the balance excludes the hydrogen, the true content is hence slightly smaller). Hence, CNCs have a sulfur molality of $m_{\mathrm{S}-\mathrm{CNC}} \approx 0.23 \mathrm{mmol}_{\mathrm{S}} / \mathrm{g}_{\mathrm{CNC}}$, and a $\sim 3 \mathrm{wt} \%$ aqueous suspensions of CNCs has a molality of $m_{\mathrm{S}-\mathrm{w}} \approx 7.0 \mu \mathrm{mol}_{\mathrm{S}} / \mathrm{mL}_{\mathrm{w}}$. The enthalpy of dissociation, $\Delta H_{\text {diss }}(T, m)$, of an ionic bond is a function of the temperature, $T$, and of the molality of the suspension, $m \cdot \frac{36}{36}$ molality of $\sim 7.0 \mu \mathrm{mol}_{\mathrm{S}} / \mathrm{mL}_{\mathrm{w}}$ may, however, be considered as an infinite dilution: $\Delta H_{\text {diss }}(T, m) \approx \Delta H^{\infty} \operatorname{diss}(T)$.

To our knowledge, there is no report of $\Delta H_{\text {diss }}(T)$ for the sulfate group of CNCs. However, the dissolution of $\mathrm{Na}_{2} \mathrm{SO}_{4}$ in water is well reported: at infinite dilutions and $298 \mathrm{~K}, \Delta H_{\text {diss }} \approx$ $-2 \mathrm{~kJ} \mathrm{~mol}^{-1} .36$ This probably represents an overestimation for CNCs since $\mathrm{Na}_{2} \mathrm{SO}_{4}$ has twice the number of cations per sulfate group. Hence, CNC's counter-ion dissociation has en enthalpy of dissociation that probably lies below $\sim-0.47 \mathrm{~J} / \mathrm{g}_{\mathrm{CNC}}$ $\left(=m_{\mathrm{S}-\mathrm{CNC}} \times \Delta H_{\text {diss }}(298 \mathrm{~K})\right)$ or about $\sim-1.3 \mathrm{~mJ} \mathrm{~m}^{-2}$ considering a CNC specific surface area of $\sim 350 \mathrm{~m}^{2} / \mathrm{g}_{\mathrm{CNC}}$. This is more than one order of magnitude below the energy variations reported in Fig. 4 for surface adsorption $\left(\sim-13\right.$ to $\left.\sim-71 \mathrm{~mJ} \mathrm{~m}^{-2}\right)$.

$$
\begin{gathered}
\mathrm{CNC}-\mathrm{O}-\mathrm{SO}_{3} \mathrm{Na} \rightleftharpoons \mathrm{CNC}-\mathrm{O}-\mathrm{SO}_{3}{ }^{-}+\mathrm{Na}^{+}, \Delta H_{\text {diss }}^{\infty}(T) \\
\mathrm{Na}_{2} \mathrm{SO}_{4} \rightleftharpoons \mathrm{SO}_{4}^{2-}+2 \mathrm{Na}^{+}, \Delta H_{\text {diss }}^{\infty}(298 \mathrm{~K}) \approx-2 \mathrm{~kJ} \mathrm{~mol}^{-1}
\end{gathered}
$$

Therefore, while the influence of counter-ion dissociation is not totally negligible, it remains secondary and should not alter the mechanisms proposed in the main text of the article. It is worth mentioning, however, that CNC surface charges have been reported to alter the interfacial stability of oil in water Pickering emulsions. $\frac{18}{18}$ It is indeed likely that electrostatic repulsion influences the maximum density of CNC particles at the oil/water interface. This work characterizes dried films whose interface is entirely made of CNCs and the influence that surface charges may have over CNC orientation during the casting process itself remains to be investigated.

\section{Wettability testing}

\section{ARCA experiments and analyses}

The video analysis of ARCA experiments returns three main parameters: the instantaneous contact angle, $\theta\left(^{\circ}\right)$, the apparent contact diameter of the drop, $D^{\text {drop }}(\mathrm{mm})$, and its volume, $V^{\text {drop }}(\mu \mathrm{L})$. Their evolution during a typical test, performed with diodomethane, is presented on Figure SI 3 . The zero-time, $t=0$, is set arbitrarily, short after the liquid enters in contact with the sample. $V^{\text {drop }}$ calculations are based on the hypothesis that the drop is axissymetric (and that its volume may thus be estimated from its cross-section). Small deviations between the calculated $V^{\text {drop }}$ value and the real amount of injected liquid may hence occur. Figure SI 3 shows four distinct phases:

- Phase I - Liquid is injected at a constant rate: $V^{\text {drop }}$ and $D^{\text {drop }}$ increase. The liquid is advancing on the surface and $\theta$ 

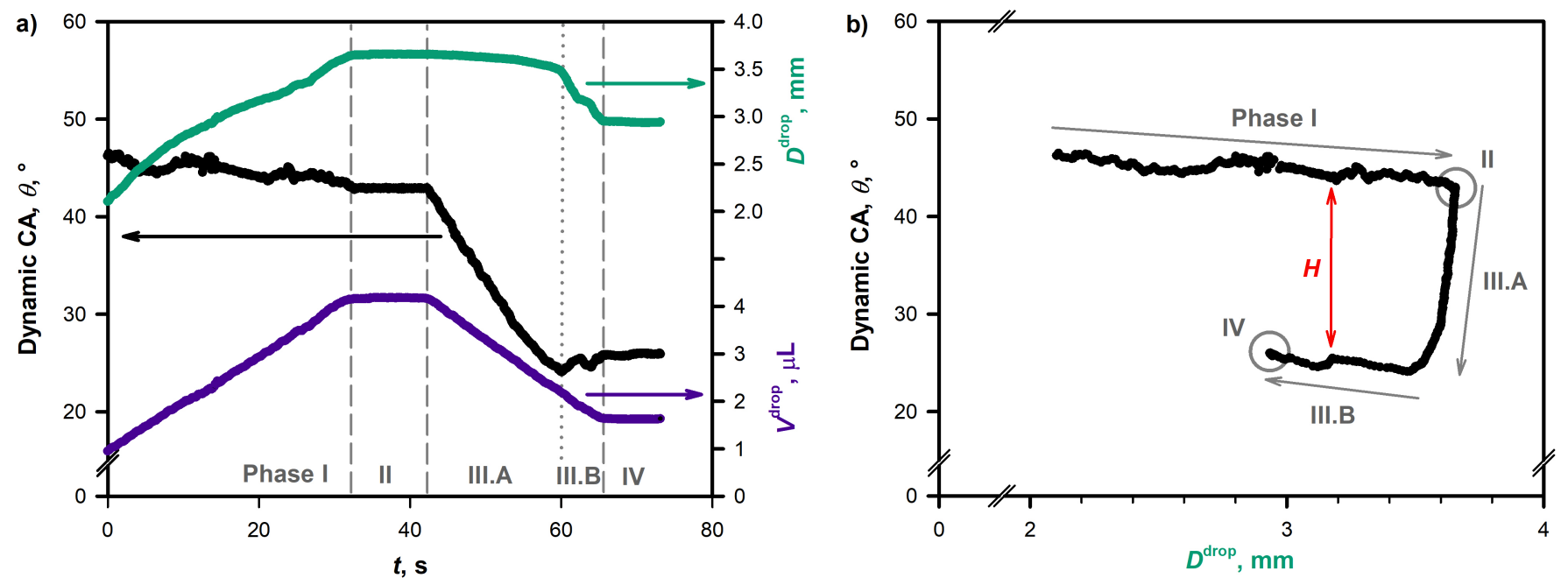

Fig. SI. 3 Evolution of the drop parameters (volume, $V^{\text {drop }}$, contact diameter, $D^{\text {drop }}$, and dynamic contact angle, $\theta$ ) over the course of an ARCA experiment conducted with diiodomethane on an oven-cast film (a). A typical experiment comprises 4 phases that correspond to liquid injection and withdrawal (phases I and III, respectively), each followed by a break of at least $5 \mathrm{~s}$ (Phases II and IV) in order to monitor the behavior of the drop at rest. In phase I, $\theta$ remains roughly constant while $D^{\text {drop }}$ grows. Phase III is subdivided between two phases: III.A during which $D^{\text {drop }}$ remains constant while $\theta$ decreases; and III.B, during which the reverse occurs. These phases are better evidenced (grey arrows) by plotting $\theta$ as a function of $D^{\text {drop }}$ (b). In this plot, all the data points corresponding to phases II and IV are superimposed (within the grey circles). This representation also highlights the magnitude of the CA hysteresis, $H$ (red arrow).

Table SI. 5 ARCA results for spin-coated and oven-cast samples analyzed with EG and DIM (Figs. 2.a\&c and in Fig. 3.a of the main text). Results are reported for each of the films (at least 3 measurements per film) and for the average of the 3 films. Standard deviations, $\sigma_{\mathrm{SD}}$, and $95 \%$ confidence intervals, $\sigma_{95 \%}$, are reported. Uncertainties reported in the main text and error bars plotted on the graphs are standard deviations.

\begin{tabular}{|c|c|c|c|c|c|c|c|c|c|c|}
\hline & \multicolumn{4}{|c|}{ Spin-coated films } & \multicolumn{4}{|c|}{ Oven-cast films } \\
\hline & & & Film 1 & Film 2 & Film 3 & SC-average & Film 1 & Film 2 & Film 3 & OC-average \\
\hline \multirow[t]{6}{*}{$\overline{\mathrm{EG}}$} & $\theta_{\mathrm{a}}$ & o & 10.1 & 10.7 & 9.7 & 10.2 & 35.6 & 45.7 & 40.1 & 41.0 \\
\hline & $\sigma_{\mathrm{SD}}$ & 。 & 0.6 & 0.4 & 0.5 & 0.6 & 0.9 & 1.7 & 2.4 & 4.3 \\
\hline & $\sigma_{95 \%}$ & $\circ$ & 1.5 & 0.9 & 1.2 & 2.8 & 2.2 & 4.3 & 3.7 & 6.6 \\
\hline & $\theta_{\mathrm{r}}$ & $\circ$ & $0^{a}$ & $0^{a}$ & $0^{a}$ & $0^{a}$ & $-b$ & $-b$ & $-b$ & $19.5^{c}$ \\
\hline & $\sigma_{\mathrm{SD}}$ & $\circ$ & - & - & - & - & - & - & - & $0.9^{c}$ \\
\hline & $\sigma_{95 \%}$ & ○ & - & - & - & - & - & - & - & $1.4^{c}$ \\
\hline \multirow[t]{6}{*}{ DIM } & $\theta_{\mathrm{a}}$ & $\circ$ & 39.4 & 32.0 & 32.9 & 34.8 & 46.7 & 46.1 & 45.6 & 46.1 \\
\hline & $\sigma_{\mathrm{SD}}$ & 。 & 0.9 & 0.9 & 1.2 & 3.4 & 0.8 & 0.7 & 1.6 & 1.2 \\
\hline & $\sigma_{95 \%}$ & ○ & 2.2 & 2.4 & 3.1 & 8.7 & 1.9 & 1.8 & 4.1 & 3.1 \\
\hline & $\theta_{\mathrm{r}}$ & $\circ$ & 13.3 & 11.0 & 10.6 & 11.6 & 24.8 & 24.8 & 24.2 & 24.6 \\
\hline & $\sigma_{\mathrm{SD}}$ & $\circ$ & 1.7 & 1.7 & 0.5 & 1.8 & 0.6 & 0.3 & 1.1 & 0.8 \\
\hline & $\sigma_{95 \%}$ & $\circ$ & 4.2 & 4.2 & 1.3 & 4.6 & 1.5 & 0.6 & 2.9 & 2.1 \\
\hline
\end{tabular}

$\overline{{ }^{a}}$ No receding for EG and the spin-coated films. ${ }^{b}$ Not enough data for a film by film breakdown. ${ }^{c}$ Based on 3 data points only (instead of 9 for the other averages). 
is thus an advancing contact angle, $\theta_{\mathrm{a}}$. Small variations in $\theta_{\mathrm{a}}$ may be attributed to surface heterogeneities, while its slight overall declining trend reflects the effect of gravity (as the drop grows, so does the Bond number, $N_{\mathrm{Bo}}$ ).

- Phase II - Liquid injection is stopped for at least $5 \mathrm{~s}$ for the drop to stabilize: $V^{\text {drop }}$ and $D^{\text {drop }}$ reach a plateau value. A constant $\theta$ during this phase indicates a proper minimization of kinetic phenomena and viscous forces. It also validates the hypothesis that the moving contact angle can be considered as being equal to the static contact angle.

- Phase III - Liquid is sucked at a constant rate: $V^{\text {drop }}$ decreases. Initially $D^{\text {drop }}$ remains constant and the loss of volume results in a decrease of the contact angle (phase III.A). This is until $\theta$ reaches a new plateau value, from which $D^{\text {drop }}$ starts to decrease. In this second part (phase III.B), the liquid is in receding conditions and a receding contact angle, $\theta_{\mathrm{r}}$, is measured. The slight increasing trend in contact angle values over time can be attributed to the reducing effect of gravity.

- Phase IV - Liquid sucking is stopped for at least $5 \mathrm{~s}$ in order for the drop to stabilize. It has the same effects and objectives than those described for the phase II.

The distinction between phases III.A and III.B is analogous to that made between the constant contact area and the constant contact angle modes during the evaporation of a sessile droplet, respectively. ${ }^{[3]}$ Plotting the contact angle as a function of the drop diameter (Fig. SI 3 b) highlights the CA hysteresis between advancing and receding conditions: $H$ is measured as the average distance (measured in ${ }^{\circ}$ for a given diameter) between the data points of the phases I and III.B (red arrow on Fig. SI 3 b). The uncertainty of the drop-based methods is commonly estimated in the range of $\pm 2^{\circ}$, to which may be added the influence of the dropsize effect. Induced by the weight of the drop, it is expected to be small at low $N_{\mathrm{Bo}}$. Nonetheless, and as evidenced in Figure SI $3 \mathrm{~b}$, the advancing CA values were found to decrease by $\sim 3-4^{\circ}$ between the moment where the droplet was small $\left(D^{\text {drop }} \approx 2 \mathrm{~mm}\right)$ and that where it became larger ( $D^{\mathrm{drop}} \approx 3.5 \mathrm{~mm}$ ). It is especially noticeable for DIM that has a high density. To enable for a comparison between the samples, experimental $\theta_{\mathrm{a}}$ and $\theta_{\mathrm{r}}$ values are hence reported as the average $\theta$ during phases I and III.B, respectively, for $D^{\mathrm{drop}}>3 \mathrm{~mm}$ ( $\sim 6$ times the diameter of the needle).

\section{Wettability results and uncertainties}

Table 5 summarizes the results plotted in Figs. 2.a\&c and in Fig. 3.a of the main text. To account for the local nature of dropbased measurements (analyses of few $\mathrm{mm}^{2}$ only), ARCA experiments were performed on at least three locations of at least three different films (minimum of 9 tests per condition). Uncertainties reported in the text and error bars plotted on the graphs are standard deviations, $\sigma_{\mathrm{SD}}$. Additionally, $95 \%$ confidence intervals, $\sigma_{95 \%}, \frac{38}{3}$ are also provided in Table 5 .

Receding measurements for EG were harder to acquire: in the the case of the spin-coated films, there was no receding at all; on the oven-cast films, most of the video footages were unexploitable and only three could be analyzed with satisfying precision. Since a film by film breakdown is impossible in these conditions, only an average CA value is reported for the receding of EG on oven-cast films and the statistical analysis was performed on 3 measurements only (instead of 9).

\section{References}

1 M. Abramoff, P. Magalhaes and S. Ram, Biophotonics International, 2004, 11, 36-42.

2 W. Y. Hamad and T. Q. Hu, Can. J. Chem. Eng., 2010, 88, 392-402.

3 G. Siqueira, J. Bras and A. Dufresne, Langmuir, 2010, 26, 402-411.

4 Q. Beuguel, J. R. Tavares, P. J. Carreau and M.-C. Heuzey, J. Colloid Interface Sci., 2018, 516, 23-33.

5 M. Kaushik, W. C. Chen, T. G. M. van de Ven and A. Moores, Nord. Pulp Pap. Res. J., 2014, 29, 77-84.

6 S. Elazzouzi-Hafraoui, Y. Nishiyama, J.-L. Putaux, L. Heux, F. Dubreuil and C. Rochas, Biomacromolecules, 2008, 9, 57-65.

7 R. J. Moon, A. Martini, J. Nairn, J. Simonsen and J. Youngblood, Chem. Soc. Rev., 2011, 40, 3941-3994.

8 R. M. Brown Jr., J. Macromol. Sci., Part A: Pure Appl. Chem., 1996, A33, 13451373.

9 S.-Y. Ding and M. E. Himmel, J. Agric. Food Chem., 2006, 54, 597-606.

10 Y. Nishiyama, P. Langan and H. Chanzy, J. Am. Chem. Soc., 2002, 124, 9074 9082.

11 G. Sèbe, F. Ham-Pichavant, E. Ibarboure, A. L. C. Koffi and P. Tingaut, Biomacromolecules, 2012, 13, 570-578.

12 C. Bruel, J. R. Tavares, P. J. Carreau and M.-C. Heuzey, Carbohydr. Polym., 2019, 205, 184-191.

13 I. Capron, O. Rojas and R. Bordes, Curr. Opin. Colloid Interface Sci., 2017, 29, 83-95.

14 L. Segal, J. J. Creely, A. E. Martin, Jr. and C. M. Conrad, Text. Res. J., 1959, 29, 786-794.

15 S. Park, J. O. Baker, M. E. Himmel, P. A. Parilla and D. K. Johnson, Biotechnol. Biofuels, 2010, 3, 10.

16 C. Bruel, T. S. Davies, J. R. Tavares, P. J. Carreau and M.-C. Heuzey, J. Colloid Interface Sci., 2020, 574, 399-409.

17 P. Scherrer, Nachrichten von der Gesellschaft der Wissenschaften zu Göttingen, Mathematisch-Physikalische Klasse, 1918, 2, 98.

18 I. Kalashnikova, H. Bizot, B. Cathala and I. Capron, Biomacromolecules, 2012, 13, 267-275.

19 M. Strobel and C. S. Lyons, Plasma Process. Polym., 2011, 8, 8-13.

20 M. Morra, E. Occhiello and F. Garbassi, Adv. Colloid Interface Sci., 1990, 32, 79-116.

21 M. Strobel, V. Jones, C. S. Lyons, M. Ulsh, M. J. Kushner, R. Dorai and M. C. Branch, Plasmas Polym., 2003, 8, 61-95.

22 C. J. van Oss, R. J. Good and R. J. Busscher, J. Dispers. Sci. Technol., 1990, 11 75-81.

23 M. S. Reid, M. Villalobos and E. D. Cranston, Nanoscale, 2016, 8, 12247-12257.

24 C. Bruel, S. Queffelou, T. Darlow, N. Virgilio, J. R. Tavares and G. S. Patience, Can. J. Chem. Eng., 2019, 97, 832-842.

25 R. G. Good, J. Adhes. Sci. Technol., 1992, 6, 1269-1302.

26 D. Y. Kwok and A. W. Neumann, Adv. Colloid Interface Sci., 1999, 81, 167-249.

27 R. G. Good, N. R. Srivatsa, M. Islam, H. T. L. Huang and C. J. V. Oss, J. Adhes Sci. Technol., 1990, 4, 607-617.

28 D. H. Kaelble, J. Adhes., 1970, 2, 66-81.

29 H. Y. Erbil and R. A. Meriç, Coll. Surf., 1988, 33, 85-97.

30 B. Jadczuk, W. Wbjcik, A. Zdziennicka and J. M. Bruque, Powder Technol., 1996, 86, 229-278.

31 W. Yu and W. Hou, J. Colloid Interface Sci., 2019, 544, 8-13.

32 C. Yamane, T. Aoyagi, M. Ago, K. Sato, K. Okajima and T. Takahashi, Polym. J., 2006, 38, 819-826.

33 Y. Peng, D. Gardner, Y. Han, Z. Cai and M. Tshabalala, J. Colloid Interface Sci., 2013, 405, 85-95.

34 V. Khoshkava and M. R. R. Kamal, Biomacromolecules, 2013, 14, 3155-3163.

35 T. Dankovich and D. Gray, J. Adhes. Sci. Technol., 2011, 25, 699-708.

36 P. Marliacy, J.-B. Bourdet, L. Schuffenecker and R. Solimando, J. Chem. Thermodyn., 2002, 34, 579-591.

37 R. G. Picknett and R. Bexon, J. Colloid Interface Sci., 1977, 61, 336.

38 D. Boffito, P. Patience, B. Srinivisan, M. Perrier and G. Patience, Can. J. Chem. Eng., 2016, 94, 1425-1430. 